\title{
A novel ditopic receptor and reversal of anion binding selectivity in the presence and absence of bound cation
}

Gamolwan Tumcharern, ${ }^{\dagger}$ Thawatchai Tuntulani, ${ }^{\dagger}$ Michael B. Hursthouse ${ }^{\ddagger}$ and Jeremy D. Kilburn

\section{Electronic supplementary information}

Contents:

Binding data from NMR titration experiments.

S1

a) NMR titration data for acetate with receptor $\mathbf{1}$ in $\mathrm{CD}_{3} \mathrm{CN} \quad \mathrm{S} 2$

b) NMR titration data for phenylphosphinate with receptor 1 in $\mathrm{CD}_{3} \mathrm{CN} \quad \mathrm{S} 5$

c) NMR titration data for diphenylphosphate with receptor 1 in $\mathrm{CD}_{3} \mathrm{CN} \quad \mathrm{S} 8$

d) NMR titration data for acetate with $\mathbf{1} \cdot \mathrm{Na}^{+}$in $\mathrm{CD}_{3} \mathrm{CN} \quad \mathrm{S} 11$

e) NMR titration data for diphenylphosphate with $\mathbf{1} \cdot \mathrm{Na}^{+}$in $\mathrm{CD}_{3} \mathrm{CN} \quad \mathrm{S} 12$

\section{Binding data from NMR titration experiments.}

The binding constants were calculated by fitting the titration data, using NMRTit HG software, kindly provided by Prof C. A. Hunter, University of Sheffield (A. P. Bisson, C. A. Hunter, J. C. Morales, K. Young, Chem. Eur. J. 1998, 4, 845). Data was recorded using standard NMR titration protocols, recording the shift for various ${ }^{1} \mathrm{H}$ signals on addition of aliquots of guest. Errors were estimated, based on quality of fit of the experimental data with the theoretical curve, and experimental errors in carrying out the experiment (concentration, volume of titres) as $<10 \%$. All association constants in the manuscript are therefore reported to 2 s.f. 
a) NMR titration data for acetate with receptor 1 in $\mathrm{CD}_{3} \underline{\mathrm{CN}}$

Solvent:

$\mathrm{CD}_{3} \mathrm{CN}$

Starting volume of host solution: $\quad 600 \mu \mathrm{L}$

Concentration of host solution: $\quad 1.87 \mathrm{mM}$

Concentration of guest solution: $\quad 18.7 \mathrm{mM}$

Association constant:

$1.11 \times 10^{4} \mathrm{M}^{-1}$

\begin{tabular}{cc}
\hline $\begin{array}{c}\text { Volume added } \\
/ \mu \mathbf{L}\end{array}$ & $\begin{array}{c}\mathbf{C H}_{\mathbf{2}} \mathbf{C H}_{\mathbf{2}} \mathbf{N H C S N H -} \\
/ \mathbf{p p m}\end{array}$ \\
\hline 0 & 7.0188 \\
5 & 7.0923 \\
10 & - \\
20 & 7.3950 \\
30 & 7.6038 \\
40 & 7.7744 \\
50 & 7.8842 \\
60 & 8.1091 \\
70 & 8.1713 \\
80 & 8.2084 \\
90 & 8.2370 \\
100 & 8.2534 \\
120 & 8.2410 \\
140 & 8.2898 \\
160 & 8.3054 \\
180 & 8.3136 \\
200 & 8.3324 \\
250 & 8.3633 \\
300 & 8.3786 \\
350 & 8.3989 \\
400 & 8.4040 \\
$\mathbf{K}_{\mathbf{a}}^{\mathbf{1 : 1}}$ & $\mathbf{1 . 1 1 \times 1 0}$ \\
\hline
\end{tabular}


Titration curves for acetate with receptor 1 in $\mathrm{CD}_{3} \mathrm{CN}$

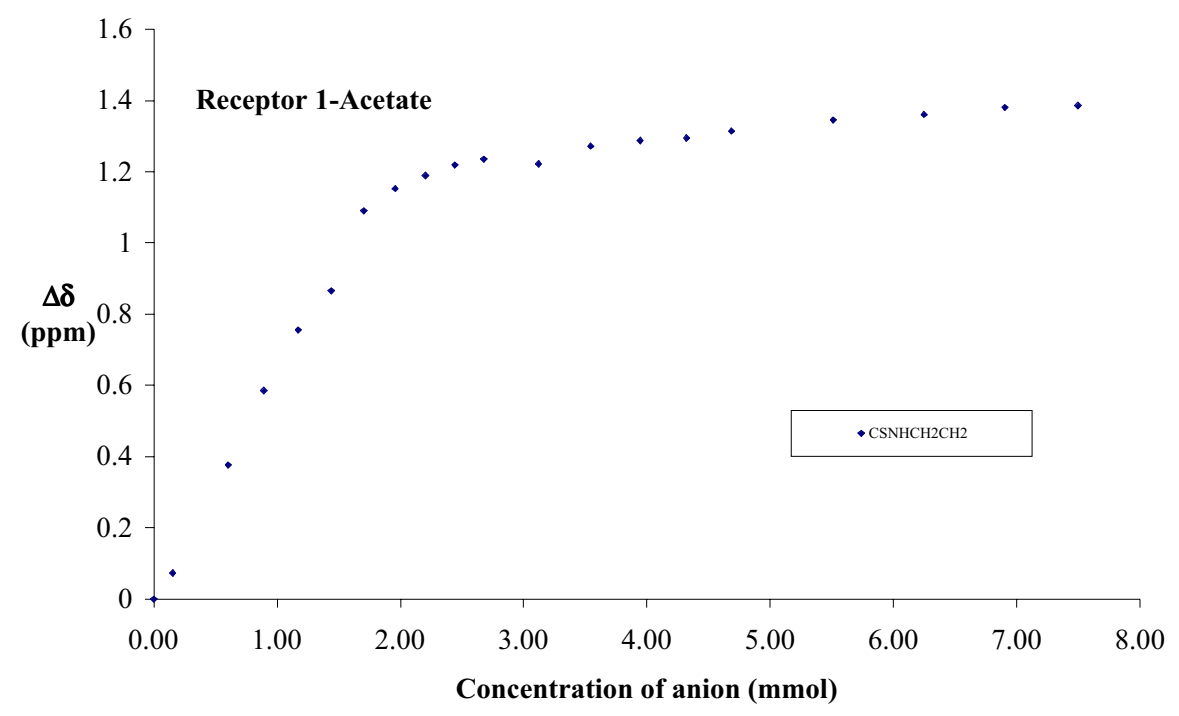

Output from NMRTit $H G$ software showing fit of theoretical curve and experimental data:

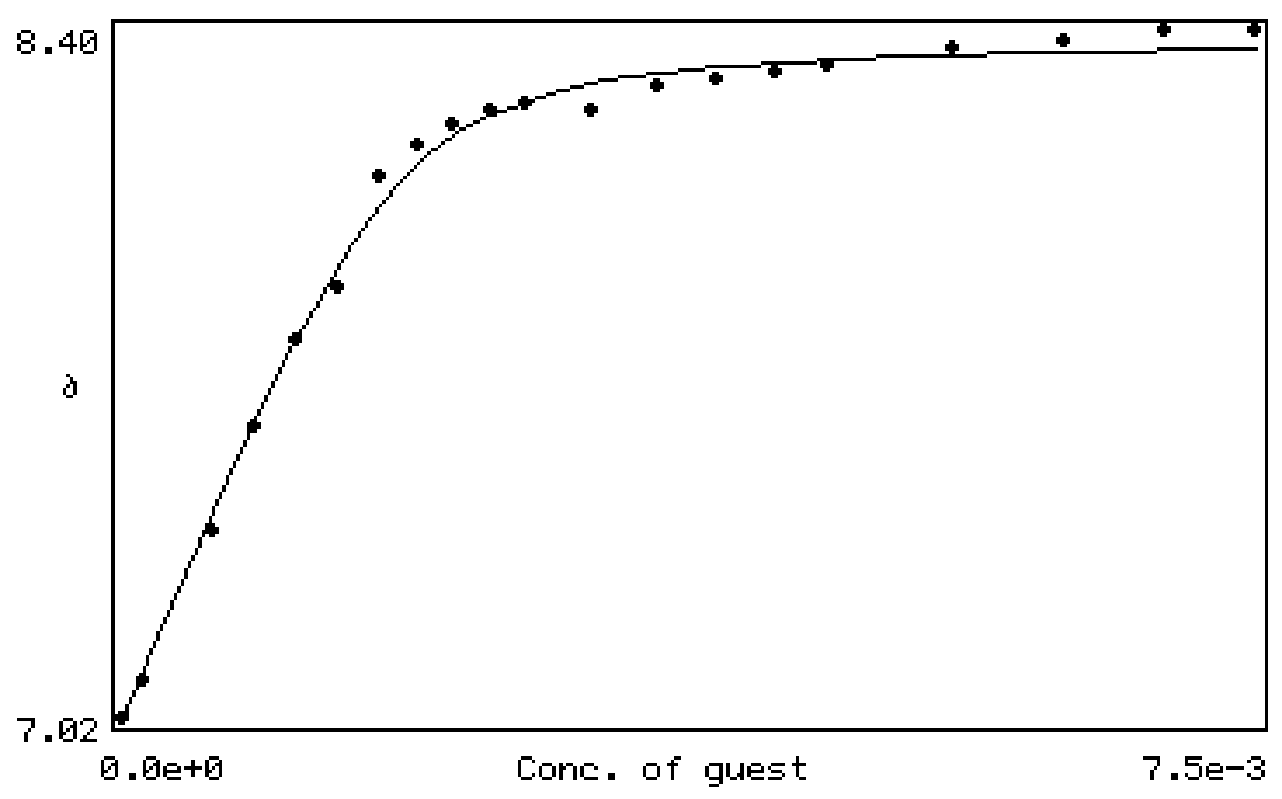


Job's plot for acetate with receptor $\mathbf{1}$ in $\mathrm{CH}_{3} \mathrm{CN}$

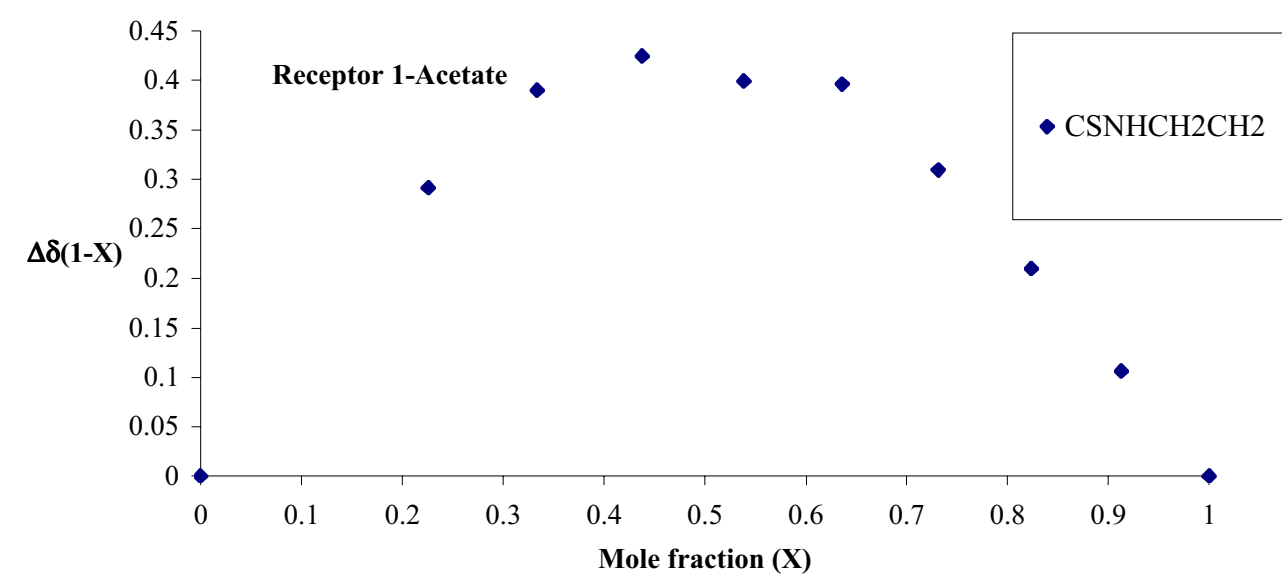


b) NMR titration data for phenylphosphinate with receptor 1 in $\mathrm{CD}_{3} \mathrm{CN}$

Solvent:

$\mathrm{CD}_{3} \mathrm{CN}$

Starting volume of host solution:

$600 \mu \mathrm{L}$

Concentration of host solution:

$1.87 \mathrm{mM}$

Concentration of guest solution:

$18.7 \mathrm{mM}$

Association constant:

$2.39 \times 10^{4} \mathrm{M}^{-1}$ (ave)

\begin{tabular}{ccc}
\hline $\begin{array}{c}\text { Volume added } \\
/ \mu \mathbf{L}\end{array}$ & $\begin{array}{c}-\mathbf{C H}_{\mathbf{2}} \mathbf{C H}_{\mathbf{2}} \mathbf{N H C S N H}- \\
/ \mathbf{p p m}\end{array}$ & $\begin{array}{c}-\mathbf{C O N H C H} \mathbf{H}^{-} \\
/ \mathbf{p p m}\end{array}$ \\
\hline 0 & 7.0024 & 7.5205 \\
5 & 7.0990 & 7.5671 \\
10 & - & 7.6007 \\
20 & 7.4157 & 7.6942 \\
30 & - & 7.8049 \\
40 & - & 7.8874 \\
50 & - & 7.9664 \\
60 & 8.1807 & 8.0328 \\
70 & 8.2910 & 8.0747 \\
80 & 8.3394 & 8.1036 \\
90 & 8.3676 & 8.1087 \\
100 & 8.3692 & 8.1220 \\
120 & 8.3899 & 8.1251 \\
140 & 8.3883 & 8.1369 \\
160 & 8.3950 & 8.1412 \\
180 & 8.4091 & 8.1467 \\
200 & 8.4208 & 8.1510 \\
250 & 8.4235 & 8.1506 \\
300 & 8.4259 & 8.1576 \\
350 & 8.4368 & 8.1693 \\
400 & 8.4364 & 8.1662 \\
$\mathbf{K}_{\mathbf{a}}^{\mathbf{1 : 1}}$ & $\mathbf{3 . 0 8 \times 1 0}$ & $\mathbf{1 . 7 0 x 1 0}$ \\
\hline & &
\end{tabular}

Titration curves for phenylphosphinate with receptor 1 in $\mathrm{CD}_{3} \mathrm{CN}$

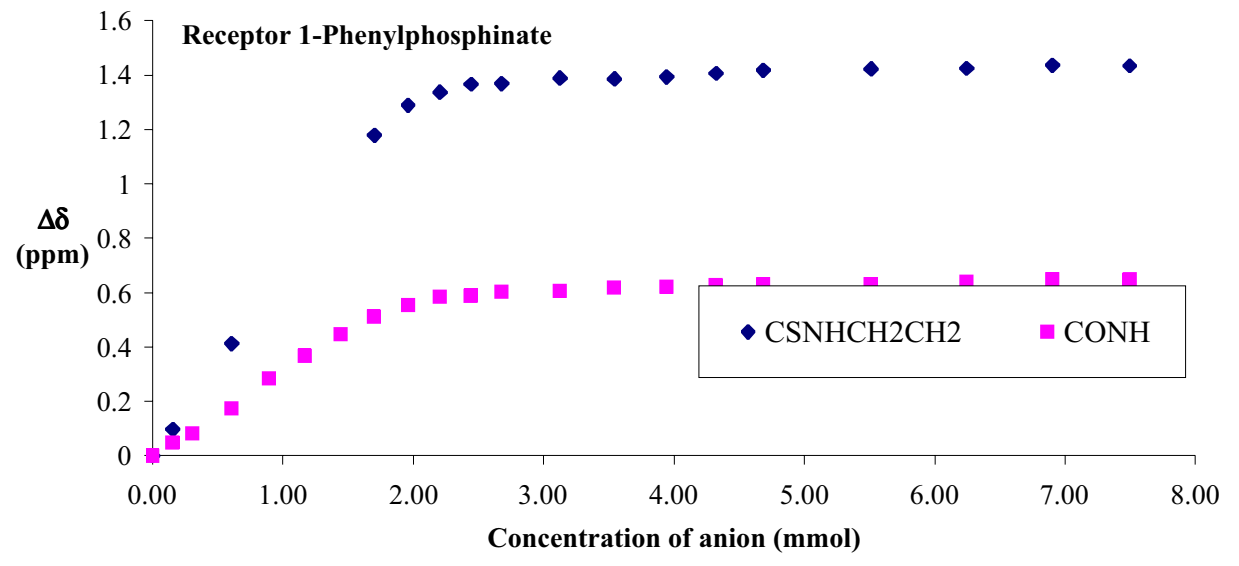


Output from NMRTit $H G$ software showing fit of theoretical curve and experimental data:

a) thiourea $\mathrm{NH}$

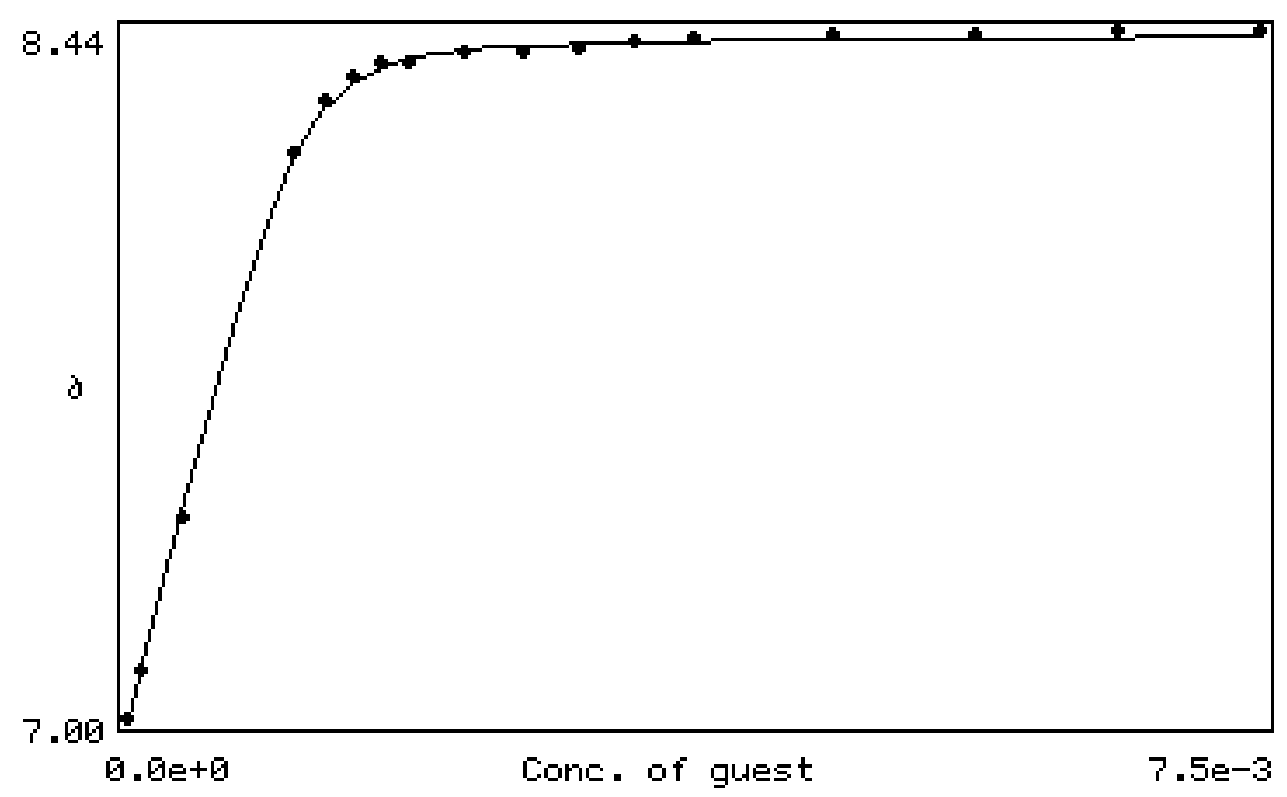

b) amide $\mathrm{NH}$

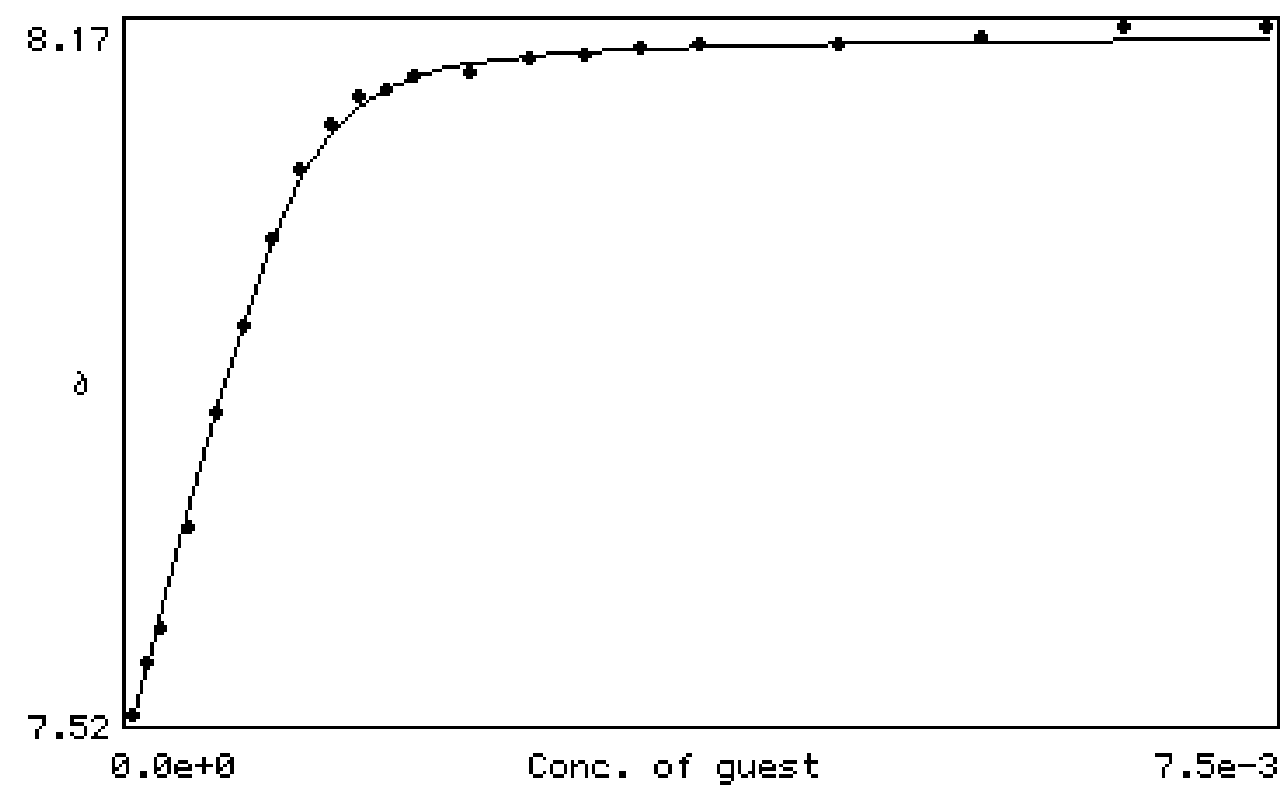


Job's plot for phenylphosphinate with receptor $\mathbf{1}$ in $\mathrm{CH}_{3} \mathrm{CN}$

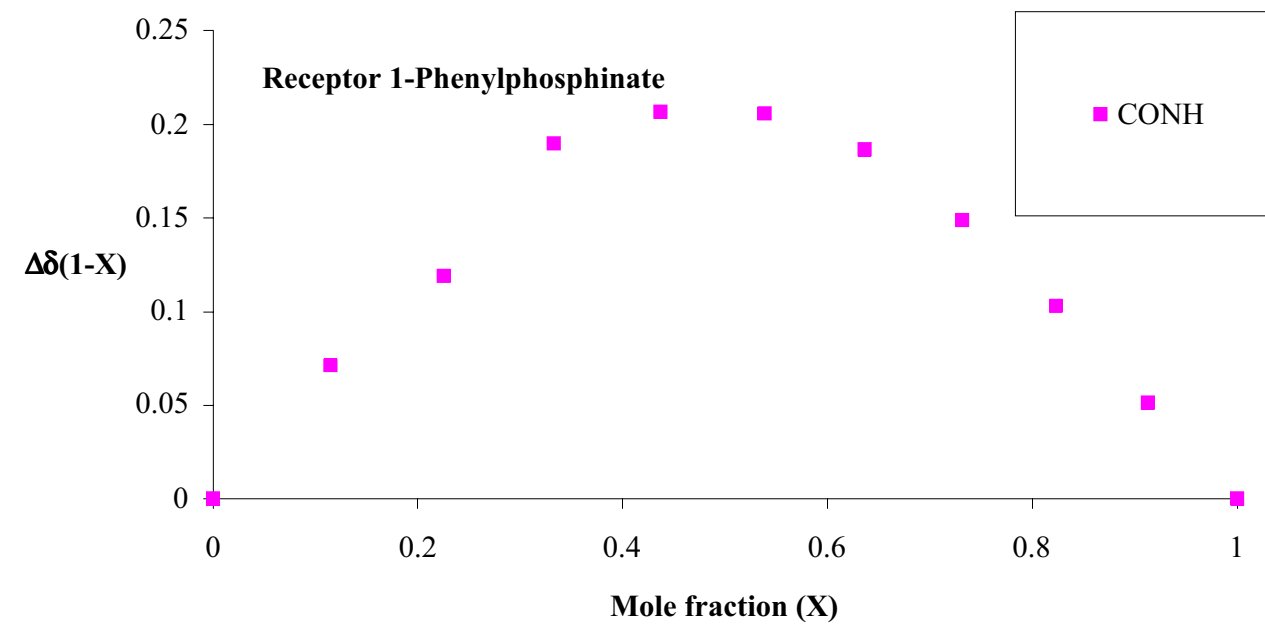


c) NMR titration data for diphenylphosphate with receptor 1 in $\mathrm{CD}_{3} \mathrm{CN}$

Solvent:

$\mathrm{CD}_{3} \mathrm{CN}$

Starting volume of host solution:

$600 \mu \mathrm{L}$

Concentration of host solution:

$1.87 \mathrm{mM}$

Concentration of guest solution:

Association constant:

$18.7 \mathrm{mM}$

$1.84 \times 10^{3} \mathrm{M}^{-1}$

\begin{tabular}{cc}
$\begin{array}{c}\text { Volume added } \\
/ \mu \mathbf{L}\end{array}$ & $\begin{array}{c}-\mathbf{C O N H C H} \mathbf{H}^{-} \\
/ \mathbf{p p m}\end{array}$ \\
\hline 0 & 7.5202 \\
5 & 7.5335 \\
10 & 7.5593 \\
20 & 7.5983 \\
30 & 7.6316 \\
40 & 7.6692 \\
50 & 7.6973 \\
60 & 7.7176 \\
70 & 7.7333 \\
80 & 7.7560 \\
90 & 7.7677 \\
100 & 7.7732 \\
120 & 7.7939 \\
140 & 7.8092 \\
160 & 7.8127 \\
180 & 7.8260 \\
200 & 7.8354 \\
250 & 7.8322 \\
300 & 7.8401 \\
350 & 7.8471 \\
400 & 7.8545 \\
$\mathbf{K}_{\mathbf{a}}^{\mathbf{1 : 1}}$ & $\mathbf{1 . 8 4 x 1 0}$ \\
& \\
\hline
\end{tabular}


Titration curves for diphenylphosphate with receptor $\mathbf{1}$ in $\mathrm{CD}_{3} \mathrm{CN}$

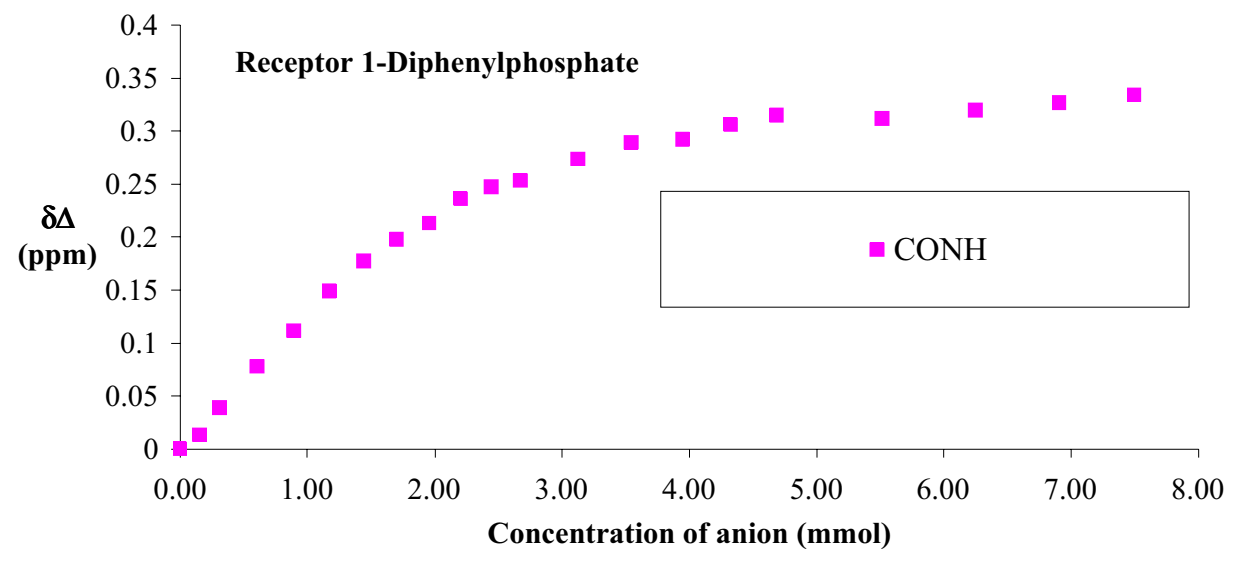

Output from NMRTit $H G$ software showing fit of theoretical curve and experimental data:

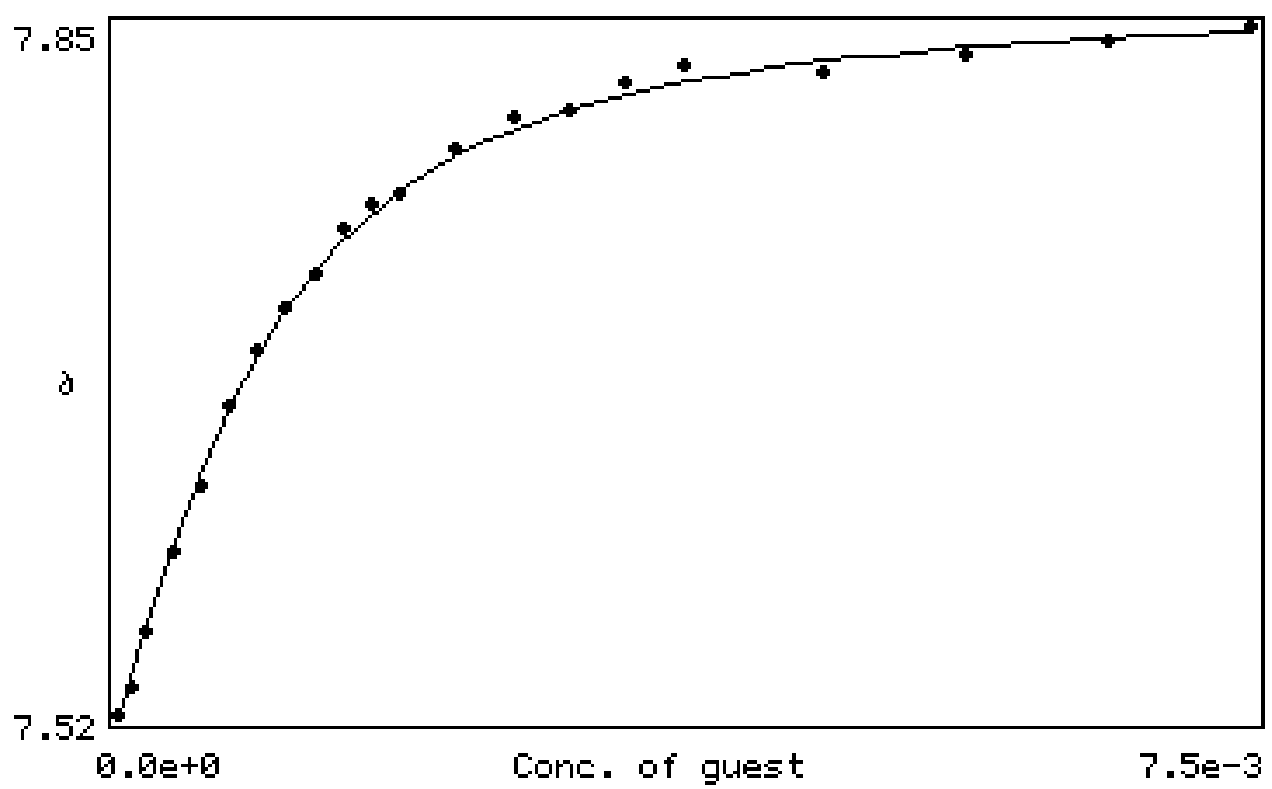


Job's plot for diphenylphosphate with receptor $\mathbf{1}$ in $\mathrm{CH}_{3} \mathrm{CN}$

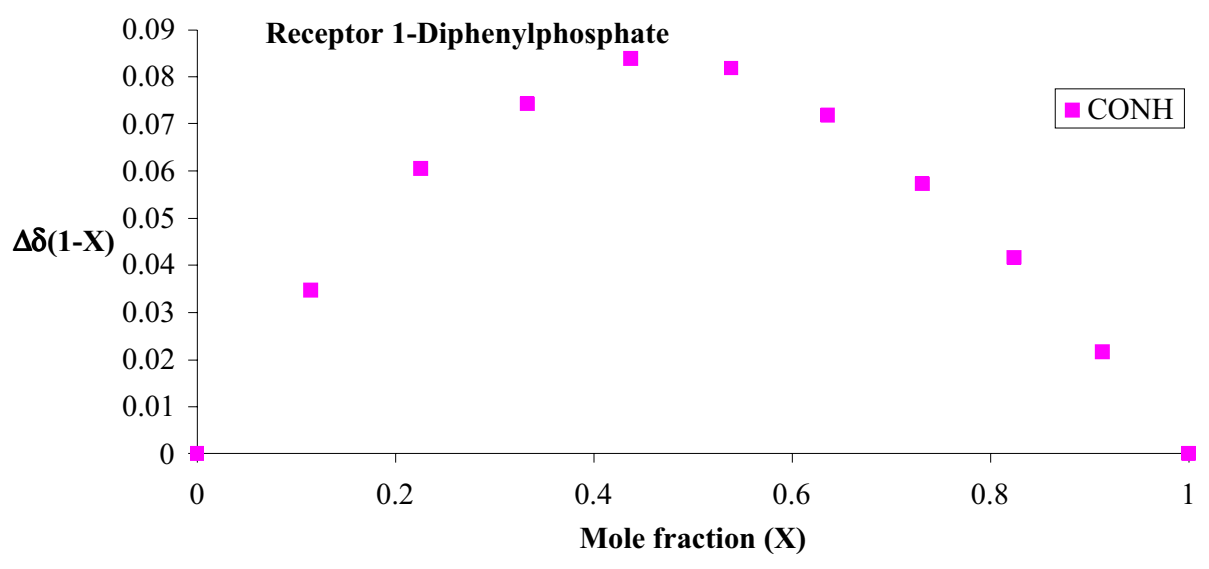




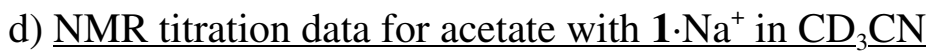

Solvent:

$\mathrm{CD}_{3} \mathrm{CN}$

Starting volume of host solution: $\quad 600 \mu \mathrm{L}$

Concentration of host solution: $\quad 1.87 \mathrm{mM}$

Concentration of guest solution: $\quad 18.7 \mathrm{mM}$

Association constant:

\begin{tabular}{ccc}
\hline $\begin{array}{c}\text { Volume added } \\
/ \mu \mathbf{L}\end{array}$ & $\begin{array}{c}-\mathbf{C H}_{\mathbf{2}} \mathbf{C H}_{\mathbf{2}} \mathbf{N H C S N H}- \\
/ \mathbf{p p m}\end{array}$ & $\begin{array}{c}-\mathbf{N H C S N H C H} \mathbf{H}_{\mathbf{2}} \mathbf{A r}- \\
/ \mathbf{p p m}\end{array}$ \\
\hline 0 & 6.9007 & 6.9007 \\
5 & 6.9623 & 6.9623 \\
10 & 7.0090 & 7.0090 \\
20 & 7.0326 & 7.0326 \\
30 & 7.0698 & 7.0698 \\
40 & 7.1107 & 7.1107 \\
50 & - & - \\
60 & - & - \\
70 & - & - \\
80 & - & - \\
90 & 7.6374 & 7.6374 \\
100 & 7.9055 & 7.9055 \\
120 & 8.2618 & 8.4023 \\
140 & 8.3961 & 8.6367 \\
160 & 8.5114 & 8.7437 \\
180 & 8.5577 & 8.8086 \\
200 & 8.5945 & 8.8533 \\
250 & 8.6454 & 8.9120 \\
300 & 8.6838 & 8.9446 \\
350 & 8.7028 & 8.9698 \\
400 & 8.7330 & 8.9855 \\
$\mathbf{K}_{\mathbf{a}}^{1: 1}$ & - & - \\
\hline
\end{tabular}

Titration curves for acetate with $\mathbf{1} \cdot \mathrm{Na}^{+}$in $\mathrm{CD}_{3} \mathrm{CN}$

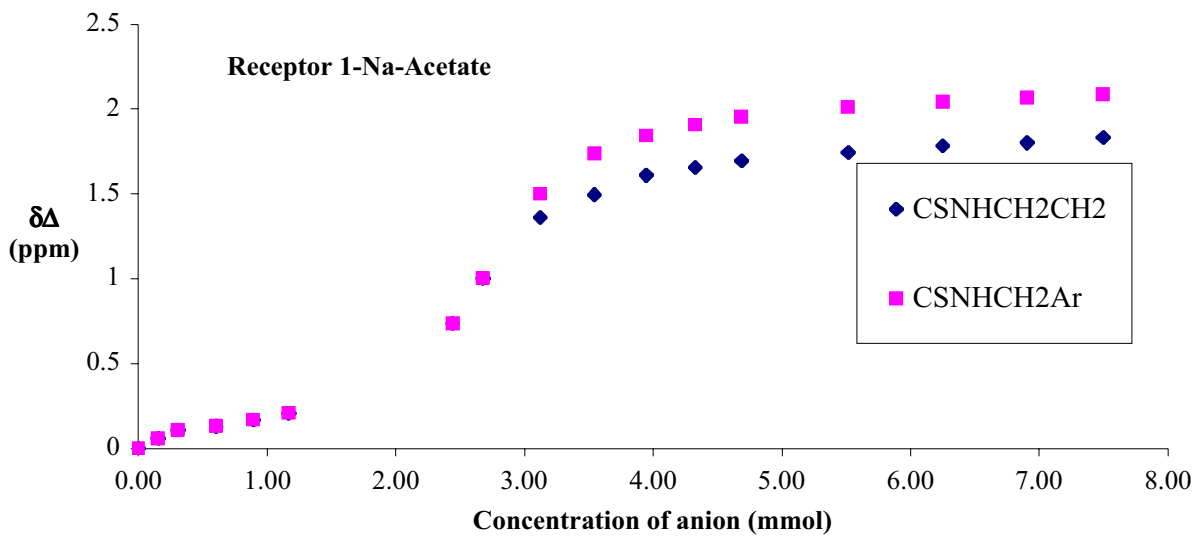


e) NMR titration data for diphenylphosphate with $1 \cdot \mathrm{Na}^{+}$in $\mathrm{CD}_{3} \underline{\mathrm{CN}}$

Solvent:

$\mathrm{CD}_{3} \mathrm{CN}$

Starting volume of host solution:

$600 \mu \mathrm{L}$

Concentration of host solution:

$1.87 \mathrm{mM}$

Concentration of guest solution:

Association constant:

$18.7 \mathrm{mM}$

$2.2 \times 10^{3} \mathrm{M}^{-1}$

\begin{tabular}{cc}
\hline $\begin{array}{c}\text { Volume added } \\
/ \mu \mathbf{L}\end{array}$ & $\begin{array}{c}\mathbf{C H}_{\mathbf{2}} \mathbf{C H}_{\mathbf{2}} \mathbf{N H C S N H -} \\
/ \mathbf{p p m}\end{array}$ \\
\hline 0 & 6.9206 \\
5 & 7.0206 \\
10 & - \\
20 & - \\
30 & 7.4968 \\
40 & 7.5266 \\
50 & 7.6014 \\
60 & 7.7214 \\
70 & 7.7662 \\
80 & 7.8472 \\
90 & 7.8931 \\
100 & 7.9524 \\
120 & 8.0315 \\
140 & 8.0437 \\
160 & 8.0756 \\
180 & 8.1042 \\
200 & 8.1052 \\
250 & 8.1262 \\
300 & 8.1448 \\
350 & 8.1504 \\
400 & 8.1603 \\
$\mathbf{K}_{\mathbf{a}}^{\mathbf{1 : 1}}$ & $\mathbf{2 . 1 5 \times 1 0 ^ { 3 }}$ \\
& \\
& \\
\hline
\end{tabular}


Titration curves for diphenylphosphate with $\mathbf{1} \cdot \mathrm{Na}^{+}$in $\mathrm{CD}_{3} \mathrm{CN}$

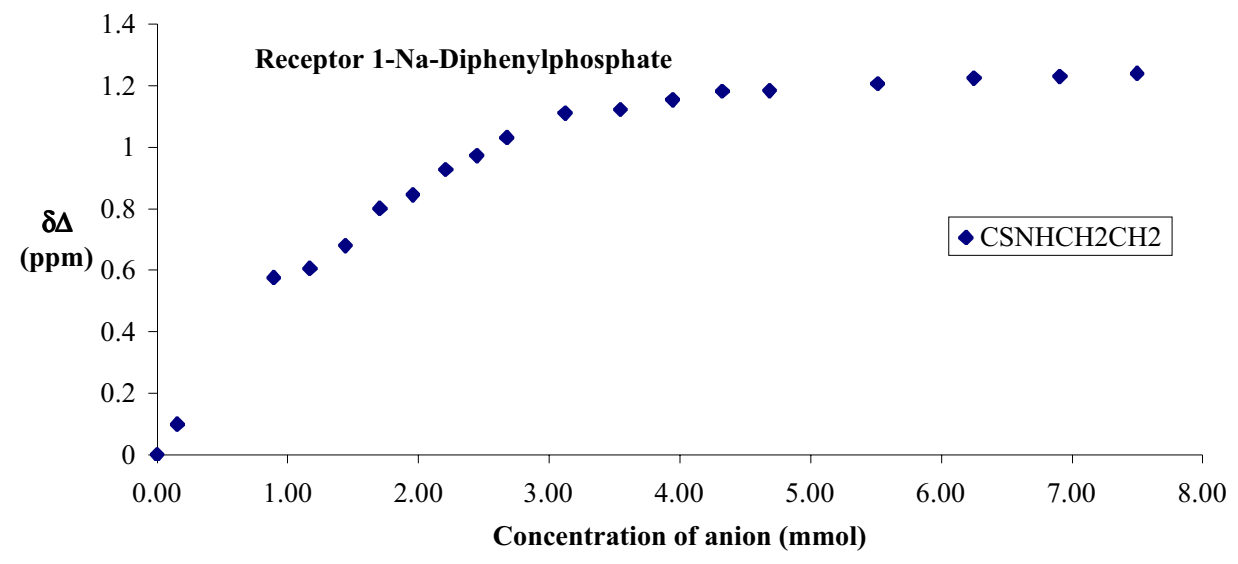

Output from NMRTit $H G$ software showing fit of theoretical curve and experimental data:

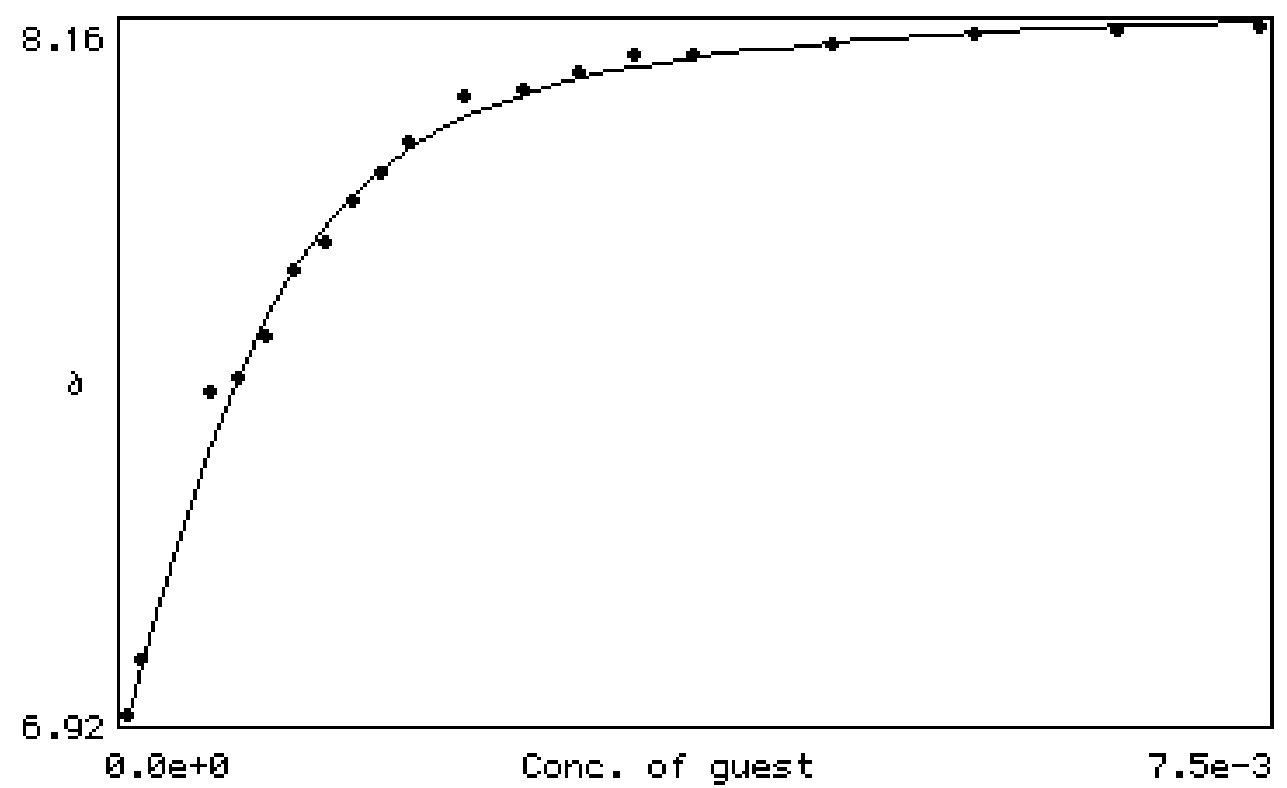

Job's plot for diphenylphosphate with $\mathbf{1} \cdot \mathrm{Na}^{+}$in $\mathrm{CH}_{3} \mathrm{CN}$

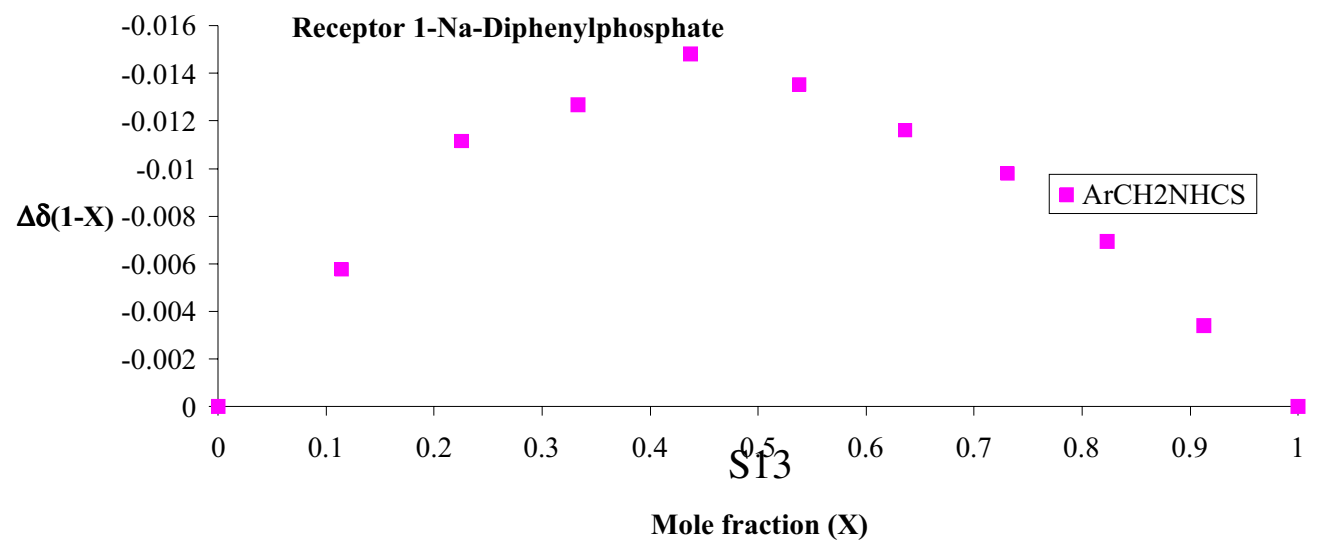

